\title{
Primary Gastric Small Cell Carcinoma (Presenting as Linitis Plastica) Diagnosed Using Endoscopic Ultrasound-Guided Biopsy: A Case Report
}

\author{
Ra Ri Cha ${ }^{1}$, Jin Kyu Cho ${ }^{2}$, Wan Soo Kim ${ }^{1}$, Jin Joo Kim ${ }^{1}$, Jae Min Lee ${ }^{1}$, Sang Soo Lee ${ }^{1}$ and Hyun Jin Kim ${ }^{1}$ \\ ${ }^{1}$ Department of Internal Medicine, Gyeongsang National University Changwon Hospital, Changwon, ${ }^{2}$ Department of Surgery, Gyeongsang \\ National University Hospital, Gyeongsang National University School of Medicine, Jinju, Korea
}

Small cell carcinomas are the most aggressive, highly malignant neuroendocrine tumors; among these, gastric small cell carcinoma (GSCC) is extremely rare. Here we report a case of a patient with primary GSCC, presenting as linitis plastic, who was diagnosed using endoscopic ultrasound (EUS)-guided biopsy. With undiagnosed linitis plastica, an 80-year-old woman was referred to our institution. Abdominal computed tomography revealed irregular wall thickening extending from the gastric body to the antrum. Endoscopy suspected to have Borrmann type IV advanced gastric cancer. EUS of the stomach showed diffuse submucosal thickening of the gastric wall, mainly the antrum. EUS-guided bite-on-bite biopsy confirmed the diagnosis of GSCC. In general, GSCC is difficult to diagnose and careful examination is necessary to determine the therapeutic strategy; however, EUS is particularly helpful in the differential diagnosis of a lesion presenting as linitis plastica. Clin Endosc 2019;52:278-282

Key Words: Extrapulmonary small cell carcinoma; Endosonography; Linitis plastica

\section{INTRODUCTION}

Primary gastric small cell carcinoma (GSCC) is a rare and the most poorly differentiated neuroendocrine tumor, accounting for less than $0.1 \%$ of all gastric cancers and $15 \%$ of all gastric neuroendocrine tumors. ${ }^{1}$ Most small cell carcinomas usually arise from the lung and rarely from the gastrointestinal tract. ${ }^{1,2}$ GSCC is diagnosed by endoscopic biopsy of the primary ulcerative or ulceroinfiltrative mass. ${ }^{3}$ Herein we report a rare case of primary GSCC presenting as linitis plastica that was diagnosed using endoscopic ultrasound (EUS)-guided biopsy.

Received: June 28, 2018 Revised: August 20, 2018

Accepted: August 23, 2018

Correspondence: Hyun Jin Kim

Department of Internal Medicine, Gyeongsang National University Changwon Hospital, 11 Samjeongja-ro, Seongsan-gu, Changwon 51472, Korea

Tel: +82-55-214-3710, Fax: +82-55-214-1036, E-mail: imdrkim@naver.com

ORCID: https://orcid.org/0000-0003-3853-0229

(c) This is an Open Access article distributed under the terms of the Creative Commons Attribution Non-Commercial License (http://creativecommons.org/ licenses/by-nc/3.0) which permits unrestricted non-commercial use, distribution and reproduction in any medium, provided the original work is properly cited.

\section{CASE REPORT}

An 80-year-old female with undiagnosed linitis plastica was referred to our institution for epigastric pain. Before visiting our hospital, she had undergone endoscopy at another hospital and was suspected of having Borrmann type IV advanced gastric cancer; however, histological examination had not confirm the malignancy. She showed epigastric tenderness after eating, which had gradually aggravated in the last month. Results of her initial physical examination were unremarkable except for epigastric pain. Her family health history was negative, whereas her medical history included medications for hypertension and diabetes mellitus over the last 10 years. Laboratory values for the serum levels of carcinoembryonic antigen and carbohydrate antigen 19-9 were within normal limits. Abdominal computed tomography of the patient revealed irregular wall thickening extending from the gastric body to the antrum, with no remarkable findings such as metastasis and infiltration of the lymph nodes or peripheral tissues (Fig. 1). No additional information was obtained from positron emission tomography-computed tomography (Fig. 2). Upper 
endoscopy showed severe mucosal fold thickening with a narrow gastric lumen and normal mucosa (Fig. 3), raising a suspicion for Borrmann type IV advanced gastric cancer. Multiple biopsies were performed; however, results of pathologic examination with endoscopic biopsy were negative. EUS (TGF-

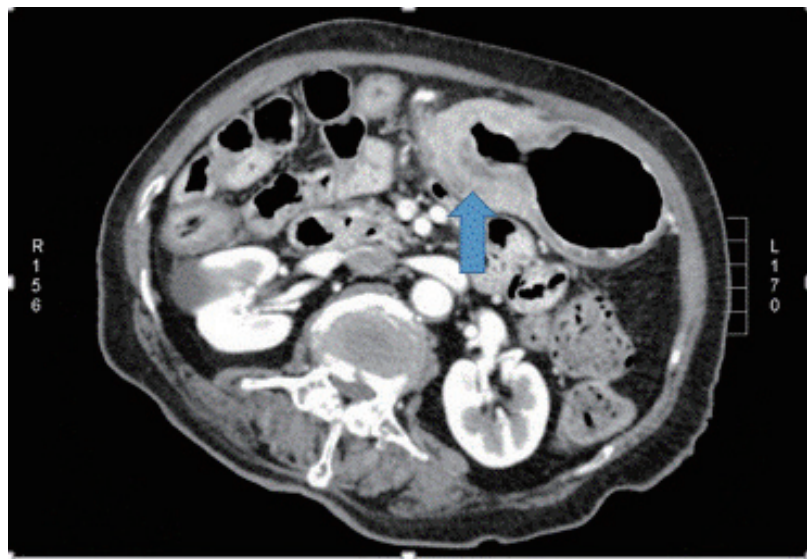

Fig. 1. Abdominal computed tomography showing diffuse thickening of the gastric wall extending from the body to the antrum (arrow).
ME1; Olympus Optical Co., Tokyo, Japan) showed normal mucosal and muscular layers and diffuse, homogeneous, and slightly hypoechoic submucosal thickening (approximately

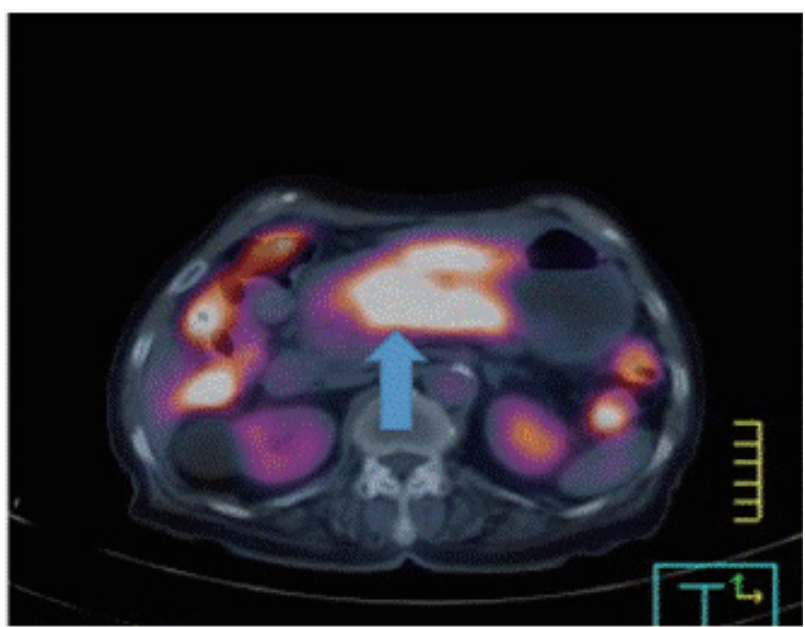

Fig. 2. Positron emission tomography-computed tomography of the chest, abdomen, and pelvis showing fluorodeoxyglucose uptake along the gastric antrum and body (arrow), with a maximum standardized uptake value of 7.76 .
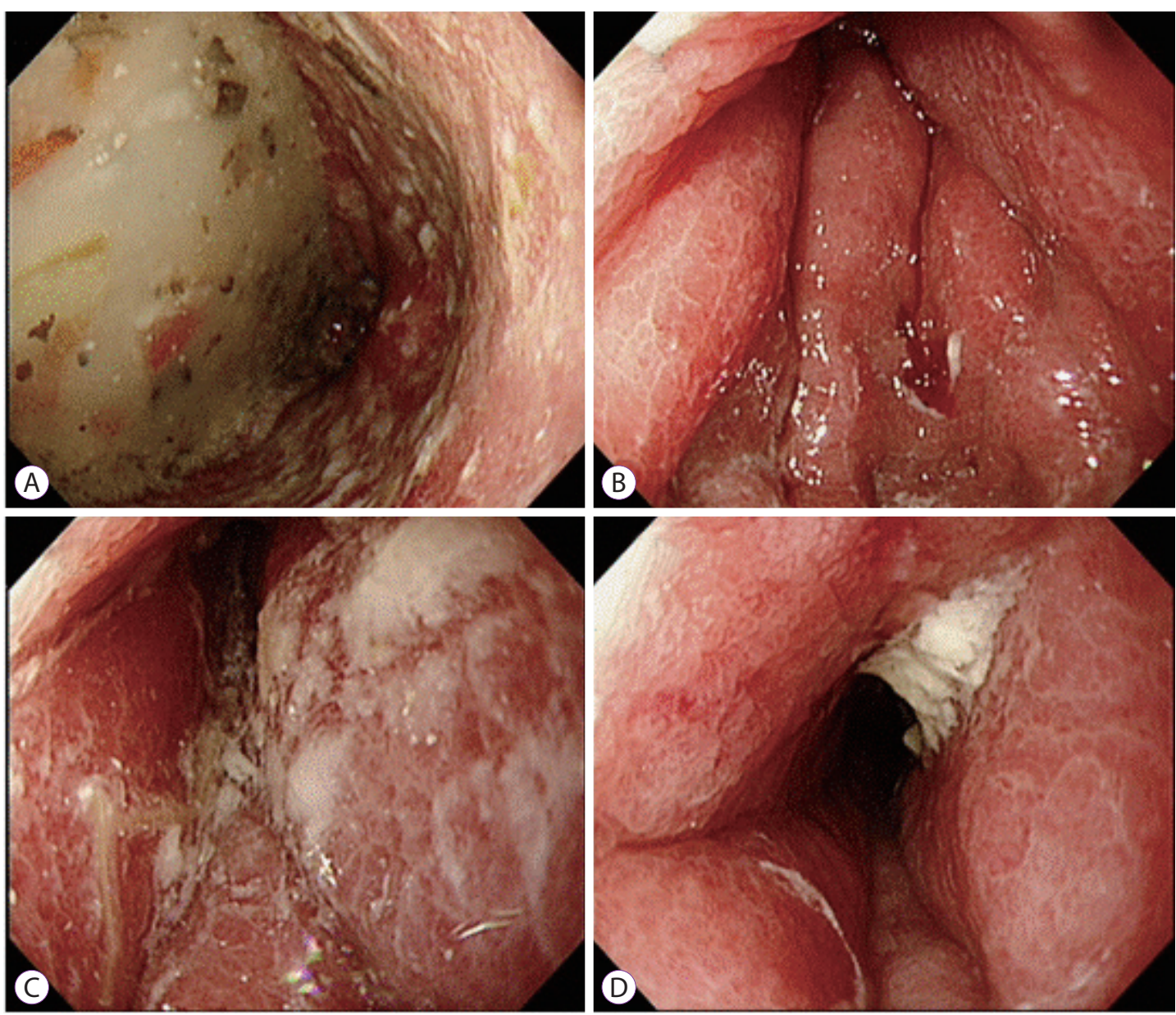

Fig. 3. Esophagogastroduodenoscopy showing diffuse circumferential thickening and poor distension of the gastric walls from the antrum to the gastric body. 
$12 \mathrm{~mm}$ thick) of the gastric wall. Although enlargement of lymph node was not observed, a small amount of ascites was detected in perigastric area (Fig. 4). Using EUS-guided biteon-bite biopsy, multiple biopsies were performed over the thickest lesion at the antrum (eight bites per lesion) to acquire the submucosal tissues. Histopathologic examination showed small, dense, uniform tumor cells with hyperchromatic nuclei and scant cytoplasm. Immunohistochemically, the tumor cells were found to be positive for cytokeratin and CD56 and negative for thyroid transcription factor-1. Ki-67 immunostaining, which reflects the proportion of highly proliferating tumor cells, revealed that $100 \%$ of tumor cells were Ki- 67 positive (Fig. 5). After the diagnosis, the patient underwent systemic chemotherapy with etoposide in combination with cisplatin, administered intravenously every 3 weeks. After the first cycle of chemotherapy, the patient was no longer able to tolerate chemotherapy owing to its adverse side effects and died due to cancer progression 3 months post-diagnosis.

\section{DISCUSSION}

Generally, the clinical course of GSCC is as aggressive as that of lung small cell carcinoma. Arai and Matsuda reported that approximately all GSCC patients die within a year of the diagnosis. ${ }^{4}$ GSCC is associated with a high incidence of lymphovascular invasion, marked deep infiltration, and distant metastases. ${ }^{5}$ Given its rarity and lack of randomized controlled trials, the standard treatment for primary GSCC has not yet been established. ${ }^{6}$

The first case of GSCC was reported in 1976 by Matsusaka et al. ${ }^{7.8}$ Generally, it is difficult to diagnose GSCC preoperatively. Kusayanagi et al. reported that only $40 \%$ of patients with GSCC are diagnosed correctly before surgery. ${ }^{9}$ Tumor cells may not be detectable in biopsy specimens because standard endoscopic biopsy sampling of submucosal infiltrating tumors often yields a false-negative result. The negative biopsy results is possible that the biopsy specimen included only the normal mucosa covering the tumor owing to the infiltration of tumor
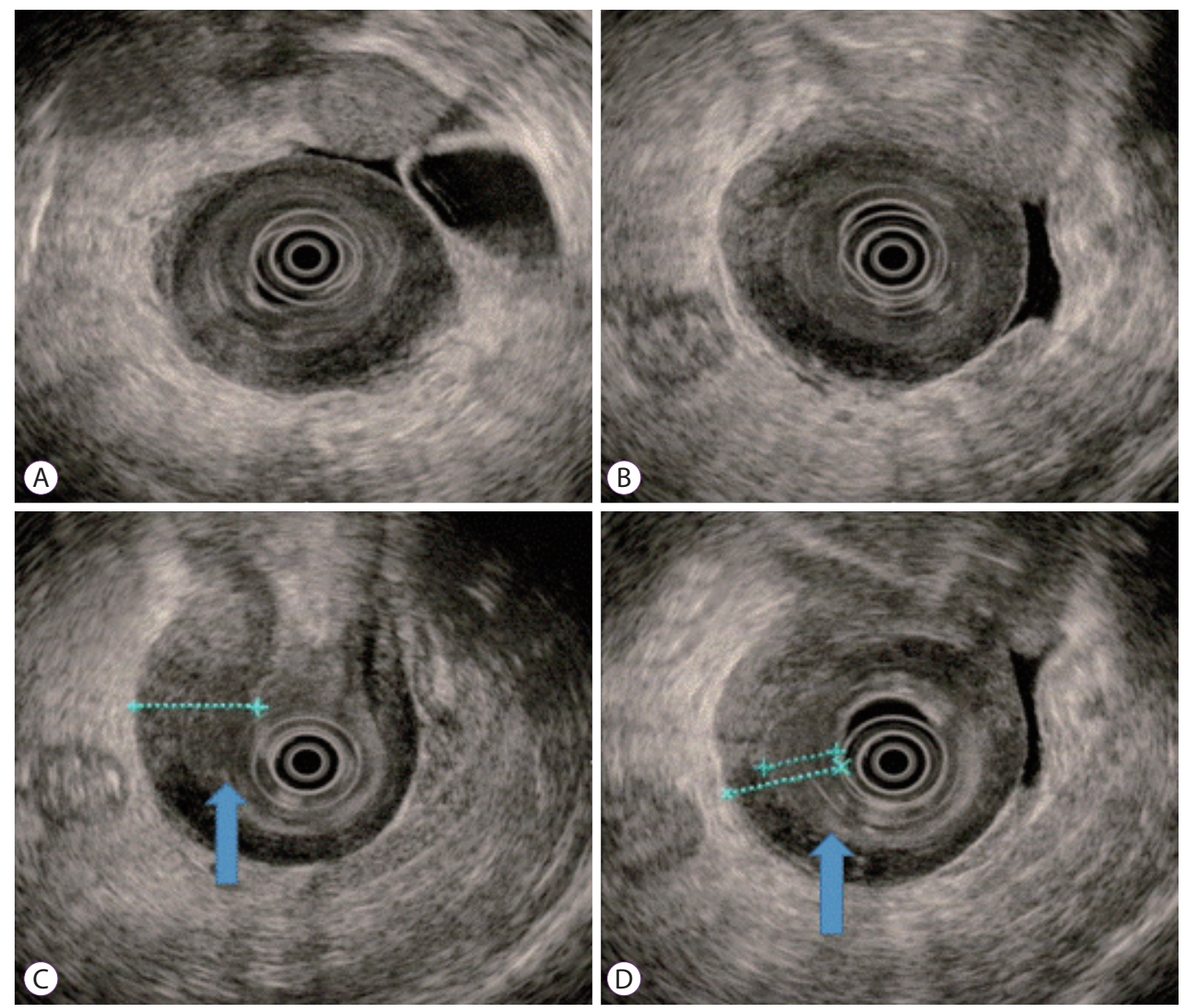

Fig. 4. Conventional endoscopic ultrasound (EUS) images. A gastric infiltrating tumor diagnosed using EUS (A, B). EUS showing homogeneous, slightly hypoechogenic thickening of the submucosal layer $(12 \mathrm{~mm})$ and intact muscularis propria layer shows reactive wall thickening $(\mathrm{C}$, arrow). The thinnest part of the mucosal lesion was selected for the biopsy after EUS localization ( $D$, arrow). 

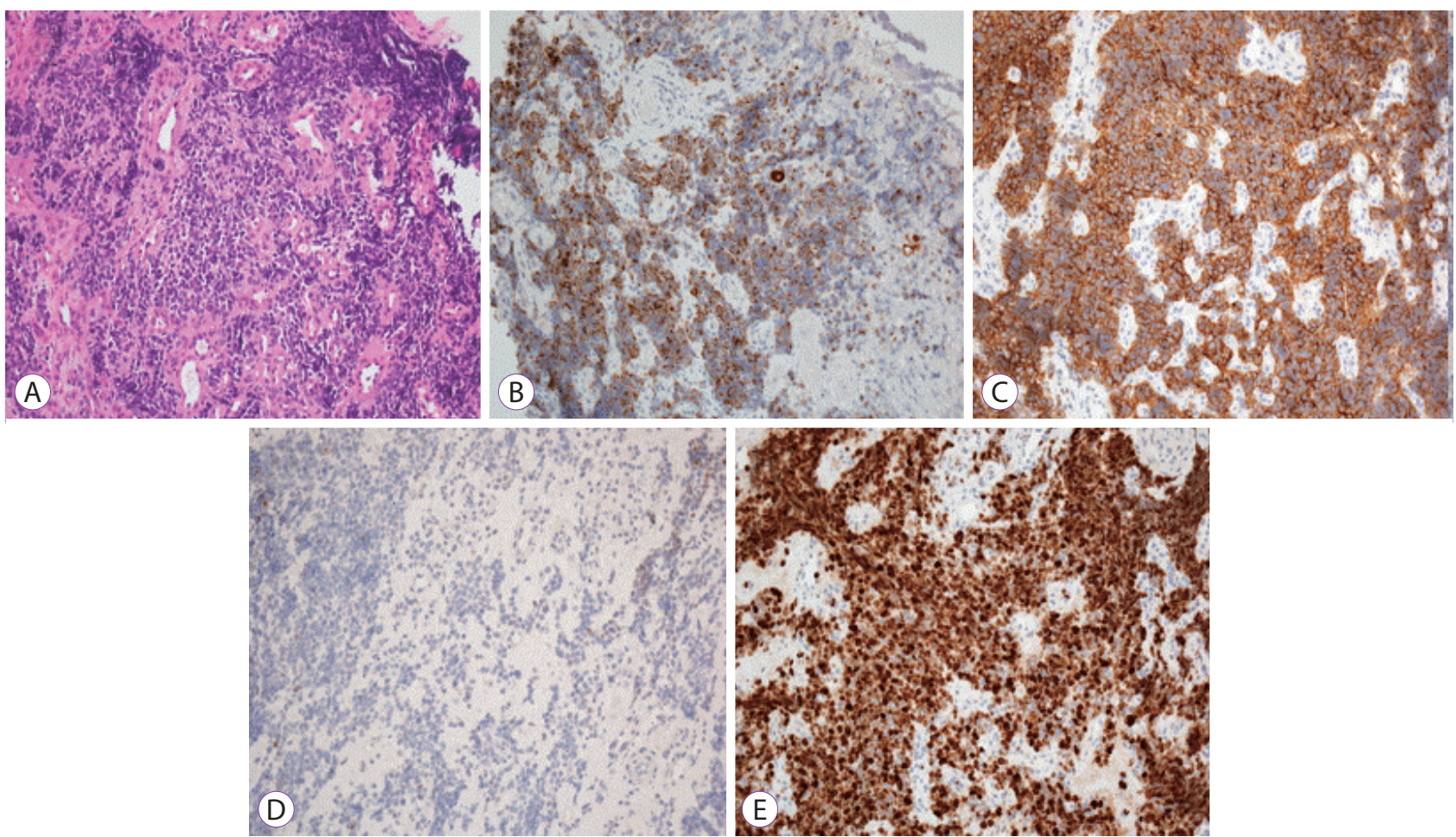

Fig. 5. Microscopic findings of the gastric tissue. Hematoxylin and eosin stained biopsy specimens show small tumor cells with irregular nuclei and scant cytoplasm (A). Tumor cells stained positive for cytokeratin (B) and CD56 (C), while they were negative for thyroid transcription factor-1 staining (D). Detection of a strong Ki-67 reactivity was observed (E). All the images were captured at $\times 200$ magnification.

cells mainly into the submucosal layer.

Linitis plastica is a type of gastric cancer characterized by poorly differentiated tumor cells that diffusely infiltrate the gastric wall, leading to reactive fibrosis. Its endoscopic characteristics include poor distension of the gastric walls and morphological changes in the gastric folds. These features make it difficult to diagnose before operation. Furthermore, though bite-on-bite biopsy is a commonly used approach for the diagnosis of linitis plastica, the diagnostic yields of the samples have not been determined. ${ }^{10}$

EUS may be an important diagnostic tool with increased diagnostic accuracy to safely detect gastric infiltrating tumors, based on their endosonographic characteristics. ${ }^{11,12}$ Furthermore, EUS is known to be helpful in diagnosing gastric linitis plastica. ${ }^{11,12}$ Under the guidance of EUS, gastric linitis plastica is generally characterized by the disappearance of five sonographic layers, which are replaced with a hypoechogenic thickening of the gastric wall, and blurring and thickening of the first three sonographic layers. ${ }^{13}$ However, in the present case, the mucosal, muscular, and muscularis propria layers were intact, and there was hypoechogenic thickening of the submucosal layer. For accurate biopsy, EUS is effective for select the optimal biopsy site. As EUS-guided bite-on-bite biopsies could obtain adequate samples, re-examination using
EUS-guided bite-on-bite technique for deep biopsy of submucosal tissues led to a correct diagnosis. Thus, we believe that EUS-guided bite-on-bite biopsy is an effective and safe diagnostic method for gastric infiltrating tumors in patients with negative biopsy results.

Immunohistochemical examination is remarkably valuable for the pathological diagnosis of GSCC. GSCC has an immunohistochemical presentation similar to that of lung small cell carcinoma, but it is unique in its positive reactions to synaptophysin, chromogranin A, neuron-specific enolase, CD56, and S-100 protein, indicating the presence of neurosecretory granules. ${ }^{14-16}$ In the current case, immunohistochemical examination showed positive expression of cytokeratin and the neuroendocrine marker CD56.

In conclusion, we report a rare case of GSCC presenting as linitis plastica that was successfully diagnosed using EUS-guided bite-on-bite biopsy. Although GSCC is difficult to diagnose before treatment, careful examination should be performed to determine the appropriate therapeutic strategy, and EUS may be particularly helpful in the differential diagnosis of a lesion presenting as linitis plastica.

\section{Conflicts of Interest}

The authors have no financial conflicts of interest. 


\section{REFERENCES}

1. Kim KO, Lee HY, Chun SH, et al. Clinical overview of extrapulmonary small cell carcinoma. J Korean Med Sci 2006;21:833-837.

2. Wu QQ, Qiang WG, Wang F, et al. Management of primary gastric small cell carcinoma in China. Int J Clin Exp Med 2015;8:1589-1597.

3. Namikawa T, Kobayashi M, Okabayashi T, et al. Primary gastric small cell carcinoma: report of a case and review of the literature. Med Mol Morphol 2005;38:256-261.

4. Arai K, Matsuda M. Gastric small-cell carcinoma in Japan: a case report and review of the literature. Am J Clin Oncol 1998;21:458-461

5. Fukuda T, Ohnishi Y, Nishimaki T, Ohtani H, Tachikawa S. Early gastric cancer of the small cell type. Am J Gastroenterol 1988;83:1176-1179.

6. Frances N, Zeichner SB, Francavilla M, Cusnir M. Gastric small-cell carcinoma found on esophagogastroduodenoscopy: a case report and literature review. Case Rep Oncol Med 2013;2013:475961.

7. Matsusaka T, Watanabe H, Enjoji M. Anaplastic carcinoma of the esophagus. Report of three cases and their histogenetic consideration. Cancer 1976;37:1352-1358.

8. Matsusaka T, Watanabe H, Enjoji M. Oat-cell carcinoma of the stomach.
Fukuoka Igaku Zasshi 1976;67:65-73.

9. Kusayanagi S, Konishi K, Miyasaka N, et al. Primary small cell carcinoma of the stomach. J Gastroenterol Hepatol 2003;18:743-747.

10. Chambers LA, Clark WE 2nd. The endoscopic diagnosis of gastroesophageal malignancy. A cytologic review. Acta Cytol 1986;30:110-114.

11. Bohle W, Scheidig A, Zoller WG. Endosonographic tumor staging for treatment decision in resectable gastric cancer. J Gastrointestin Liver Dis 2011;20:135-139.

12. Vetro C, Chiarenza A, Romano A, et al. Prognostic assessment and treatment of primary gastric lymphomas: how endoscopic ultrasonography can help in tailoring patient management. Clin Lymphoma Myeloma Leuk 2014;14:179-185.

13. Songur $\mathrm{Y}$, Okai T, Watanabe $\mathrm{H}$, et al. Endosonographic features of a calcified mucinous gastric carcinoma. Endoscopy 1995;27:347.

14. Matsui T, Kataoka M, Sugita Y, et al. A case of small cell carcinoma of the stomach. Hepatogastroenterology 1997;44:156-160.

15. Brenner B, Tang LH, Klimstra DS, Kelsen DP. Small-cell carcinomas of the gastrointestinal tract: a review. J Clin Oncol 2004;22:2730-2739.

16. Nakamura Y, Otani S, Otaka M, et al. Gastric small cell carcinoma with marked response to neoadjuvant chemotherapy. Int J Clin Oncol 2005;10:348-352. 